\title{
Detrimental cross-talk between sepsis and acute kidney injury: new pathogenic mechanisms, early biomarkers and targeted therapies
}

\author{
Sergio Dellepiane ${ }^{1 \dagger}$, Marita Marengo ${ }^{2 \dagger}$ and Vincenzo Cantaluppi ${ }^{3^{*+}}$
}

\begin{abstract}
This article is one of ten reviews selected from the Annual Update in Intensive Care and Emergency medicine 2016. Other selected articles can be found online at http://www.biomedcentral.com/collections/ annualupdate2016. Further information about the Annual Update in Intensive Care and Emergency Medicine is available from http://www.springer.com/ series/8901.
\end{abstract}

\section{Background}

Acute kidney injury (AKI) complicates about $3-50 \%$ of hospital admissions [1] depending on patient comorbidities and on medical procedures performed during the stay. The greatest incidence of AKI is observed in the intensive care unit (ICU): indeed, critically ill patients with hospital-acquired AKI have a greater in-hospital mortality (30-70\%) and a more than double risk of severe adverse outcomes even 5 years after discharge, including an increased incidence of end-stage chronic kidney disease (CKD), than patients without AKI [2]. The most common cause of in-hospital AKI is sepsis, the systemic inflammatory response to infection that is often complicated by multiple organ failure and death [3]. In recent years, experimental and clinical studies have provided new insights into the pathogenic mechanisms of sepsisassociated AKI, also explaining how the loss of renal function may impair the immune system and lead to the subsequent development of sepsis. In this chapter, we

\footnotetext{
* Correspondence: vincenzo.cantaluppi@med.uniupo.it

${ }^{\dagger}$ Equal contributors

${ }^{3}$ Department of Translational Medicine, University of Eastern Piedmont,

"Maggiore della Carità" University Hospital, Novara, Italy

Full list of author information is available at the end of the article
}

review the recent findings on the detrimental cross-talk between sepsis and AKI and the identification of new, early biomarkers and targeted therapies aimed at improving the outcome of these critically ill patients.

\section{New pathogenic mechanisms of sepsis-associated aki: the 'liaison dangereuse' revisited}

Major advances have been made in our understanding of the detrimental connection between the systemic inflammatory response to infection and the acute loss of kidney function with consequent improvements in clinical practice. In particular, recent findings have challenged the dogma that in the course of severe sepsis and septic shock, AKI is merely a consequence of ischemic damage due to tissue hypoperfusion (Fig. 1).

\section{Renal overflow rather than hypoperfusion}

According to old theories, tissue hypoperfusion associated with sepsis causes renal ischemia and consequently acute tubular necrosis. By contrast, AKI is also found in the early phases of severe sepsis even in absence of an impaired cardiac output and in milder infectious diseases without manifest systemic signs. In a large prospective study including more than 1800 patients, Murugan et al. reported that AKI was frequent in patients with non-severe pneumonia, including those not transferred to the ICU and without hemodynamic instability [3]. In addition, the few studies reporting data on biopsies or autopsies from patients who developed sepsis-associated AKI have demonstrated that tubular necrosis is not common. Moreover, the number of apoptotic cells is significantly lower than that observed in any other types of AKI and not related to the severity of renal dysfunction [4]. On this basis, it is now accepted that septic AKI is only in part sustained by renal hypoperfusion. Di Giantomasso and coworkers elegantly 


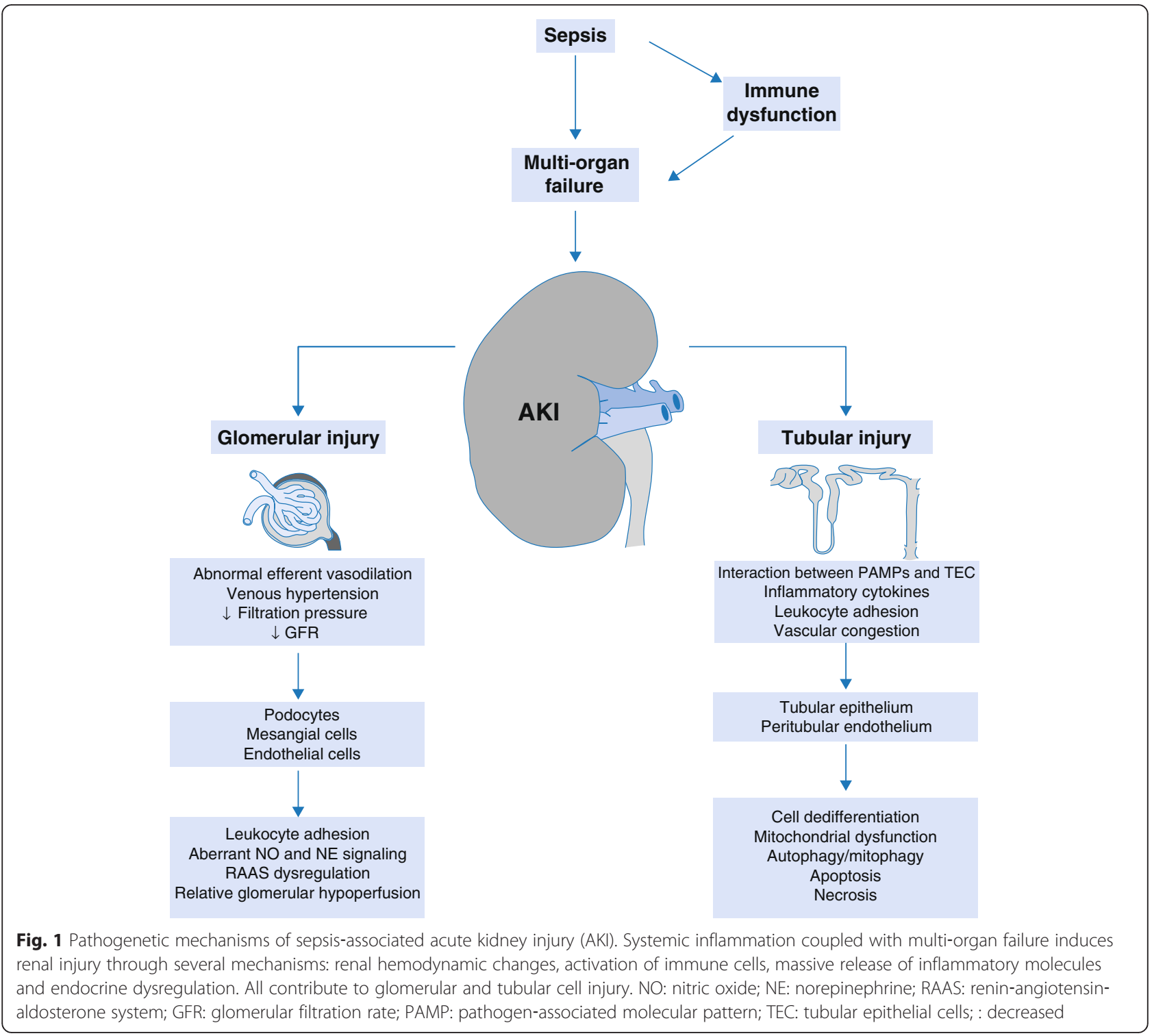

proved this new theory in a sepsis model of sheep subjected to invasive monitoring of renal blood flow (RBF) [5]. Interestingly, these authors found that RBF was normal or even increased in sepsis and proposed the new model of hyperdynamic septic AKI [5]. Furthermore, increased RBF in septic AKI has also been observed in humans using thermodilution and magnetic resonance imaging (MRI) [6]. As a proof of concept, other studies have confirmed in vitro that plasma obtained from patients with sepsis-associated AKI induced tubular epithelial cell dysfunction without the contribution of any ischemia-reperfusion injury [7].

Based on these data, septic AKI is currently considered to be the consequence of several concomitant factors: a dysfunction of the renal microvascular system; direct interaction of pathogen fragments with renal resident cells; the cytotoxic effect of the sepsis-induced cytokine storm; and, finally, the deleterious cross-talk between injured organs. All these changes are sustained by fascinating intracellular mechanisms that may be targeted by new therapeutic approaches.

\section{Microvascular and glomerular changes in septic AKI}

The parenchymal distribution of blood flow during sepsis is still far from being completely understood: the only incontestable point is the concomitant reduction in the glomerular filtration rate (GFR). For this reason, several studies have focused on the mechanisms that decouple RBF from GFR. It has been shown that sepsis triggers a redistribution of $\mathrm{RBF}$ that leads to relative cortical hypoperfusion and to medullary overflow: this effect is enhanced by administration of norepinephrine [8]. 
Nevertheless, peritubular flow has been found to be sluggish and congested, probably as the result of a high capacity and low resistance circulation [9]. Other theories about the GFR decline during sepsis have focused on the reduction of filtration pressure not necessarily associated with glomerular hypoperfusion; renal and central (CVP) venous pressures are frequently increased in sepsis as a possible consequence of the so-called fluid challenge of septic patients in the first hours after ICU admission. This relative venous hypertension may induce microvascular congestion and tissue edema, thus affecting GFR. In support of this hypothesis, a retrospective analysis of 105 ICU patients found a linear correlation between the CVP and AKI incidence or duration [10]. Moreover, it has been shown that fluid overload in septic patients is independently associated with death and worse renal outcomes [11]. Another theory is based on the presence of efferent arteriole over-dilation as a consequence of altered angiotensin signaling or regional differences in nitric oxide (NO) and norepinephrine production and/or sensitivity [8]. The role of norepinephrine seems particularly relevant within the renal parenchyma: preliminary clinical and experimental studies have reported that the use of sympatholytic agents, such as clonidine or the $\$ \backslash$ upalpha-2 receptor antagonist, dexmedetomidine, improves outcomes in septic AKI [12]. It has also been proposed that inflammationactivated shunt systems may directly connect afferent and efferent arterioles, thus decreasing GFR. However, although this effect is present in experimental studies in large animals subjected to sepsis, it has not been yet recognized in humans [8]. Finally, tubular cell dysfunction (see next paragraph) may induce the back-leakage of filtered substances and a resultant GFR decrease completely independent from glomerular physiology.

\section{Systemic inflammation directly affects tubular cell function}

Sepsis comprises the concomitant presence of an invasive infection and a host systemic inflammatory response syndrome (SIRS). SIRS is characterized by an over-inflammatory state due to a massive and deregulated activation of innate andadaptive immunity usually followed by an equally massive and deleterious counterregulatory response leading to the so-called 'immune paralysis'. Accordingly, a bi-modal trend in patient mortality has been described with a first early phase due to SIRS and a second late phase caused by immunosuppression and lymphocyte exhaustion [13].

As stated above, renal inflammatory injury may be consequent to the direct interaction of resident cells with pathogens as well as with molecules released following the deregulated host immune response. Different pathogen-associated molecular patterns (PAMPs), such as lipopolysaccharide (LPS), lipoteichoic acid and porins, may directly interact with resident kidney cells. In particular, tubular epithelial cells express Toll-like receptor 4 (TLR4), the molecule with the highest affinity for LPS. It has been shown that LPS reduces the expression of the endocytic receptors megalin and cubilin in the apical compartment of proximal tubular epithelial cells, leading to an interference with the normal processes of protein re-absorption, thus contributing to the typical low molecular weight proteinuria of septic patients [7].

Renal resident endothelial and tubular cells directly communicate with the immune system: indeed, these cells not only express cytokine receptors, but they are also able to release several inflammatory proteins and to increase the expression of specific adhesion molecules able to recruit circulating cells that perpetuate tissue damage. It has also been demonstrated that tubular epithelial cells can recruit $\mathrm{T}$ cells by expressing vascular cell adhesion molecules (VCAMs) and intercellular cell adhesion molecules (ICAMs) and that they are consequently able to activate lymphocytes via the co-stimulatory molecule CD40 [14].

Circulating inflammatory cytokines directly affect the renal parenchyma and are associated with an increased risk of mortality in AKI patients [15]. The prominent role of cytokines in septic patients was further highlighted by a recent randomized controlled trial based on the administration of afelimomab (an antitumor necrosis factor [TNF]- $\alpha$ monoclonal antibody). Patients with higher basal levels of interleukin (IL)-6 had a small but significant mortality reduction when treated with this monoclonal antibody and, although the authors did not focus on AKI incidence, a significant improvement in the overall sequential organ failure assessment (SOFA) score was observed $48 \mathrm{~h}$ after the infusion [16]. Of interest, some of these inflammatory mediators, such as TNF- $\alpha$, IL-6, CD40-ligand and Fas-ligand, can directly interact with specific counter-receptors located on tubular epithelial cells causing loss of function and apoptotic cell death.

A further mechanism of renal injury in the course of sepsis is probably linked to detrimental organ crosstalk. Apart from the validated models of hepatorenal and cardiorenal syndromes, recent studies have highlighted the effect of lung, brain and bone marrow as sources of potential nephrotoxic molecules. Indeed, studies performed in vivo and in vitro using plasma derived from patients treated with mechanical invasive ventilation showed that pneumocytes produce IL- $1 \beta$, IL- 6 , IL- 8 and TNF- $\alpha$ after ventilator-induced biotrauma and that the release of these cytokines promoted tubular cell apoptosis and consequently AKI. Moreover, the massive cytokine release observed after traumatic brain injury is a putative further cause of tubular cell dysfunction and has been 
associated with renal injury and consequent delayed graft function after donation for kidney transplantation [14]. The mechanisms of interaction between systemic inflammation and tubular epithelial cells and the potential clinical consequences of sepsis-associated AKI are shown in detail in Fig. 2.

\section{Mechanisms of cellular dysfunction}

All the hemodynamic and cytotoxic alterations described in the previous paragraphs directly affect kidney cell survival and function. Several types of cellular injury occur in the course of AKI, including necrosis, apoptosis or their combined form, necroptosis. This latter type of cellular injury is a highly immunogenic form of programmed cell death that normally represents a defense against viruses expressing caspase- 8 inhibitors, but may also be triggered by cytokine imbalance [14].
The role of this pathway in AKI has not been completely investigated.

As noted earlier, at biopsy or autopsy, septic patients have a significantly lower rate of tubular apoptosis than other ICU patients with AKI. Furthermore, the amount of necrosis also seems to be marginal. In addition, both apoptosis and necrosis are found in a few limited parenchymal areas and do not correlate with renal dysfunction and AKI duration. The direct consequence of these observations is that septic AKI is probably associated with a series of cell dysfunctions rather than cell death. Available histological data have demonstrated mostly tubular cell vacuolization and mitochondrial rarefaction, possible consequences of autophagy and mitophagy, respectively. Based on these findings, it has been proposed that tubular epithelial cells react to injury by regressing to a dedifferentiated and energy-saving state. Accordingly, it has

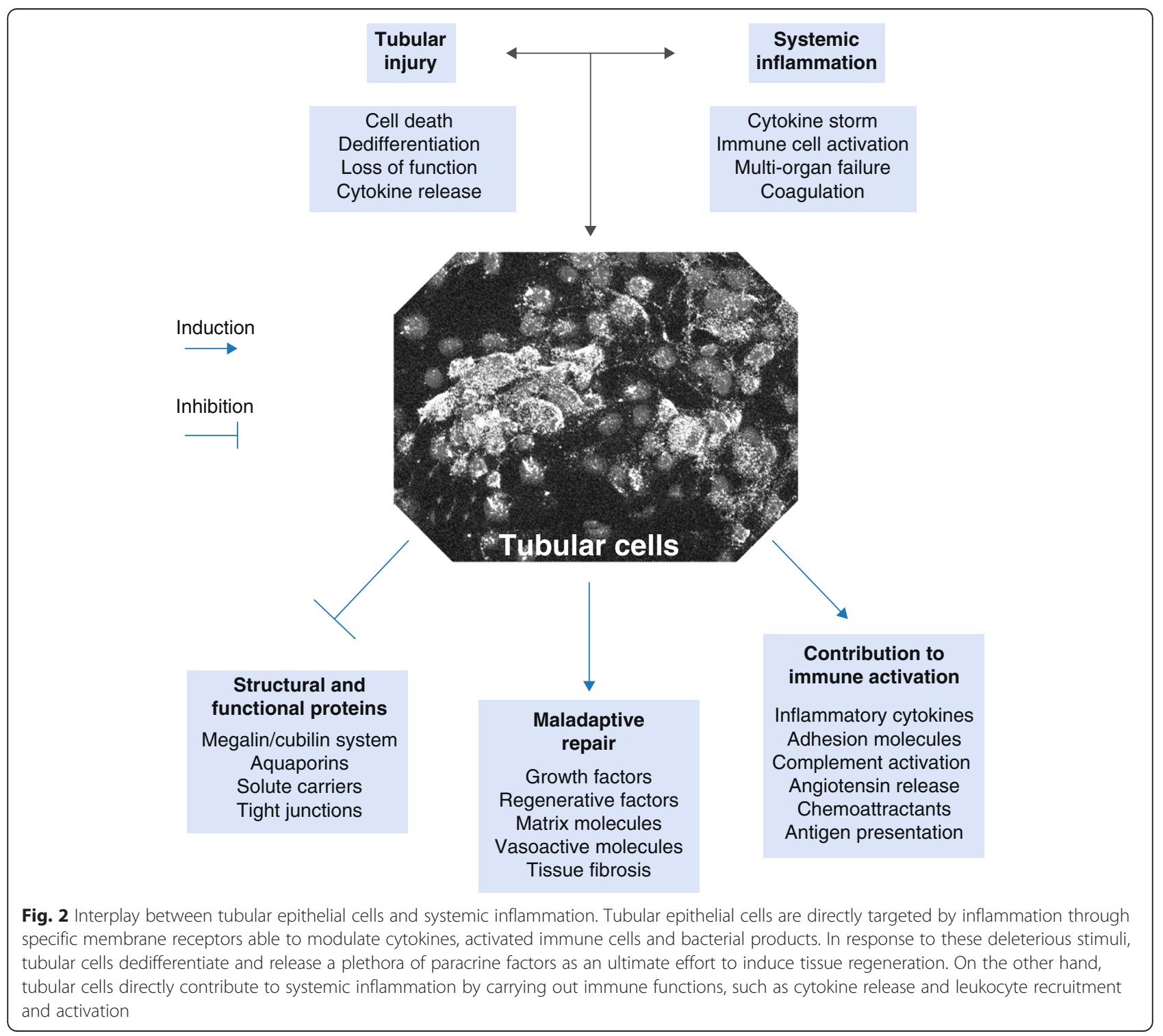


been found that during sepsis, renal oxygen consumption is significantly reduced [17]. The auto-digestion of energy-consuming organelles (autophagy), including the mitochondria (mitophagy), is one of the key passages of this biological phenomenon. Moreover, it has been shown that tubular cells exposed to septic plasma lose cell polarity and the expression of the endocytic receptor, megalin [7]. These alterations confirmed the transition of tubular cells toward a dedifferentiated state with the consequent loss of preservation of two distinct fluidfilled compartments characterized by precise electrolyte concentrations. As a consequence of tubular dysfunction, both electrolyte reabsorption and protein trafficking are almost completely abolished during septic AKI [7].

Another energy-sparing strategy is the arrest of the cell cycle. This phenomenon has been demonstrated in tubular cells subjected to a septic microenvironment. Accordingly, two of the most promising urine early biomarkers of AKI (tissue inhibitor of metalloproteinases [TIMP]-2 and insulin-like growth factor-binding protein [IGFBP]-7) are proteins involved in the G1-S phase transition arrest [18]. A further mechanism of tubular dysfunction may depend on the horizontal cross signaling between proximal and distal segments. Kalakeche et al. reported that cellular uptake of LPS in the S1 segment caused oxidative stress at both S2 and S3 levels [19].

Finally, recent data suggest that AKI may contribute to the development and aggravation of a systemic inflammatory state. Indeed, tubular cells are immunologically active, because they are able to act as antigen-presenting cells and are devoted to the clearance of various soluble mediators involved in inflammation. These findings may at least in part explain the pathogenic mechanisms underlying the accelerating development of sepsis and multiple organ failure when patients lose tubular cell function. In this setting, Naito et al. elegantly proved the direct influence of LPS on tubular cell gene expression. These authors demonstrated in vivo increased RNApolymerase II density at TNF- $\alpha$, monocyte chemotactic protein-1 (MCP-1) and heme oxygenase-1 (HO-1) loci after LPS injection in mice. This effect was related to selective histone methylation and was enhanced by different nephrotoxic stimuli [20]. These AKI-associated epigenetic alterations of tubular cells may be responsible for the above-mentioned increase in circulating inflammatory mediators.

\section{Biomarkers of sepsis-associated AKI}

AKI is currently defined using the Risk, Injury, Failure, Loss of kidney function, and End-stage kidney disease (RIFLE), Acute Kidney Injury Network (AKIN) and Kidney Disease - Improving Global Outcomes (KDIGO) criteria [21]: all these systems are based on the levels of serum creatinine (SCr), estimated GFR (eGFR) and on urine output. However, these parameters are insensitive, non-specific and have a substantial latency, in particular in patients with sepsis-associated AKI. Indeed, sepsis per se is known to decrease creatinine production; moreover, the fluid overload that frequently characterizes septic patients may be responsible for a further delay in $\mathrm{SCr}$ increase. Urine output is also influenced by several confounding factors: its rapid decrease not associated with changes in GFR is currently classified as 'transient' or 'subclinical' AKI, with uncertain clinical significance and not consistent with the presence of parenchymal injury [22].

Several molecules have recently been proposed for the early diagnosis of sepsis-associated AKI. However, numerous problems confound the validation of these molecules in the clinical setting. First, these biomarkers are always compared to the above-mentioned defective classifications. Moreover, the current scarcity of effective therapies limits the effect of early diagnosis on the achievement of hard clinical endpoints. These two aspects concur to underestimate the diagnostic accuracy of these new identified molecules. Finally, some of the AKI biomarkers are (or are functionally associated with) acute phase proteins and are influenced by the inflammatory state. On this basis, the cytokine storm observed during sepsis may lead to a false-positive interpretation. However, despite these limits, several studies have demonstrated substantial diagnostic improvements using different molecules.

Neutrophil gelatinase-associated lipocalin (NGAL) is a $25 \mathrm{kDa}$ protein belonging to the lipocalins, a class of soluble factors involved in small molecule traffic. NGAL binds to prokaryotic and eukaryotic siderophora, ironchelating molecules involved in bacterial growth and tissue differentiation, respectively. During the course of AKI, NGAL is increased in both serum and urine. Serum NGAL is mainly produced by hepatocytes and immune cells, whereas urinary NGAL derives in part from serum NGAL filtered by glomeruli and in part from NGAL released by kidney tubular epithelial cells following injury. NGAL is the most extensively studied biomarker in septic AKI and is also frequently used as the control molecule when other proteins are investigated. NGAL has a bimodal trend with a first peak before and a second peak 24-48 h after AKI onset [23]. A limitation of clinical NGAL use is that its increase in both serum and urine may be related to the presence of a systemic inflammatory state including sepsis and not only to the development of AKI. Indeed, it has been shown that patients developing only sepsis or only AKI have similar serum and urinary NGAL levels: by contrast, non-AKI nonseptic patients and those affected by both clinical conditions are clearly classified [21]. To summarize these studies, urinary and serum NGAL levels are 
more associated with sepsis severity (and the presence of multiple organ failure) than with AKI incidence. To reduce this limitation, some authors propose to strengthen NGAL performance by combining it with sepsis-specific biomarkers. Lentini et al. measured circulating levels of NGAL together with advanced oxidation protein products (AOPP) in 98 consecutive ICU patients. The authors found that NGAL values were slightly higher in patients with only AKI than in those with only sepsis without any significant difference: however, AOPP levels were able to distinguish between septic and non-septic subjects and allowed a correct classification for most patients. Similar results were obtained coupling serum NGAL levels with endotoxin activity assay (EAA) results, which can identify LPSinduced neutrophilic responses in Gram-negative bacteria, septic shock [21]. Despite these encouraging data, a key point for NGAL measurement still remains unresolved: three different forms of NGAL have been isolated - a $25 \mathrm{kDa}$ monomer, a $45 \mathrm{kDa}$ dimer and a $135 \mathrm{kDa}$ heterodimer, conjugated with gelatinase. Currently, no commercially available assays can discriminate between the monomer, mainly released from tubular epithelial cells, and the dimer, originating from neutrophils [22]. Future development in this context may further increase the diagnostic accuracy of this molecule.

Triggering receptor expressed on myeloid cells (TREM)-1 is a membrane receptor belonging to the immunoglobulin superfamily and expressed on neutrophils and monocytes. Its soluble form, sTREM, is massively released in body fluids during sepsis, pneumonia, septic arthritis, meningitis, peritonitis, and uterine cavity infections. In septic patients, urinary sTREM is increased $24-48 \mathrm{~h}$ before clinical evidence of AKI, whereas a continuous increase in STREM independently correlates with sepsis progression and with a worse outcome [24]. However, similar to NGAL, sTREM correlates with AKI as well as with systemic inflammation and more studies are needed to detect possible confounding factors.

The host SIRS is the cornerstone of sepsis development: SIRS is caused by an immune system over-reaction to pathogens and induces a 'cytokine storm'. Additionally, recent findings have demonstrated that several cytokines are involved in AKI development and some are directly produced by renal cells. In a cohort of ICU patients, Cho et al. found that IL-8 and IL-10 were increased in AKI patients independent of the presence of sepsis. Interestingly, the authors found a significant increase in soluble CD25 (IL-2 receptor) in septic AKI [15]. Soluble CD25 is a marker of T regulatory cell activity and it may correlate with the development of a counter-regulatory response leading to immune paralysis.
The combined use of urinary IGFBP-7 and TIMP-2 has recently been approved by the FDA as an early indicator of AKI [18]. Other new biomarkers of AKI include liver fatty acid binding protein (L-FABP), IL-18, netrin-1, kidney injury molecule-1 (KIM-1) and $\alpha 1$-microglobulin. Other circulating mediators, including CD40-ligand, Fas-ligand, angiopoietin-2 and presepsin (soluble CD14) have been validated in septic patients as indicators of mortality $[21,25]$. In the next few years, the evolving field of 'omics' technologies (genomics, transcriptomics, proteomics, and metabolomics) may lead to a system biologybased approach that could improve diagnostic strategies for sepsis-associated AKI.

\section{Targeted therapeutic approaches for sepsis- associated AKI}

Several clinical trials in patients with severe sepsis and septic shock have failed to show an improvement in outcomes. Despite the initial benefits of so-called early goaldirected therapies, a prolonged series of therapeutic failures of promising strategies including corticosteroids, activated protein $\mathrm{C}$ and the use of standard renal replacement therapies (RRT) has been observed. Septic patients still have an unacceptable high mortality and, excluding antimicrobial agents, clinical management is almost exclusively based on supportive therapies that are not able to interfere with the underlying pathogenic mechanisms of concomitant tissue damage and immunoparalysis. In this section, we summarize the more recent advances in the therapeutic treatment of experimental and clinical sepsis-associated AKI.

\section{Pharmacological agents}

The severity of injury and the poor outcomes associated with septic AKI worsen with delayed recognition of renal dysfunction. Early identification of AKI in septic patients is crucial because employed supportive and therapeutic strategies are frequently nephrotoxic (e.g., antibiotics, such as vancomycin and aminoglycosides, use of vasopressor therapy without adequate fluid resuscitation, etc.) and may additionally worsen the extent of the renal injury. A recent clinical trial by Sood et al. [26] showed that septic patients who had reversible or improved AKI within $24 \mathrm{~h}$ of diagnosis had better survival rates than patients who did not recover from AKI and even than those who did not develop AKI at all. Most of the available interventions for AKI are based on prevention of further renal insult or organ support (early administration of appropriate antimicrobial therapy, restoration of tissue perfusion and optimization of the hemodynamic status), but these standard treatment approaches have not been effective in significantly reducing the incidence of septic AKI. Furthermore, the emergence of antibioticresistant microbes as well as the increased clinical 
complexity of patients emphasizes the need to understand and to develop novel pharmacologic approaches to successfully treat sepsis-associated AKI.

The paradigm of sepsis-associated organ dysfunction and AKI has focused largely on specific cytokines and their modulation or removal for improving outcome. However, therapeutic approaches based on targeting specific pathways known to contribute to the pathogenesis of septic AKI have failed. Indeed, clinical trials focused on antagonizing a single detrimental mediator including anti-endotoxin (LPS), TNF- $\alpha$, IL$1 \beta$, and TLR-4 did not show any significant results [27]. These failures may be, at least in part, explained by the early-deregulated host immune response mediated by the activation of innate immunity followed by a state of immunosuppression. For this reason, therapeutic approaches should consider the temporal profile of the immune status in sepsis: the early blockade of pro-inflammatory pathways should be followed by distinct therapies aimed at triggering the late activation of immunity. On this basis, Swaminathan et al. recently reviewed emerging therapeutic approaches in septic AKI, focusing on targeting early pro-inflammatory and late anti-inflammatory processes [28]. The suppression of early inflammation during sepsis-associated AKI could be obtained by using alkaline phosphatase. This enzyme is able to reduce inflammation through dephosphorylation and thereby 'detoxification' of LPS, which is a key mediator of sepsis-induced organ failure, including AKI. Furthermore, alkaline phosphatase catalyzes the conversion of adenosine triphosphate into adenosine, a potent anti-inflammatory factor. The administration of recombinant alkaline phosphatase reduced inflammation and the incidence of septic AKI, but no changes in mortality rate were observed [29]. In order to attenuate the early inflammatory response, some authors have also proposed the use of cannabinoid 2 (CB2) receptor agonists [30]. CB2 receptors are expressed on leukocytes and regulate the immune system: their activation attenuates leukocyte-endothelial cell interaction and the recruitment of leukocytes, thus reducing the amount of circulating pro-inflammatory mediators. Fibrates, which are peroxisome proliferator-activated receptor- $\alpha$ (PPAR $\alpha)$ agonists, have been shown to ameliorate sepsis induced by Gram-negative bacteria by promoting neutrophil recruitment [28]. Experimental studies recently described the role of the cholinergic anti-inflammatory pathway: stimulation of the vagus nerve may attenuate cytokine release in sepsis, ischemia-reperfusion injury and other states of inflammation [31]. Moreover, nicotine (an $\alpha 7$ nicotinic acetylcholine receptor agonist) has been shown to reduce mortality in sepsis [28]. Other studies have demonstrated that soluble thrombomodulin was effective in preventing and treating established AKI, an effect associated with reduced leukostasis and endothelial cell permeability [32].

Later in the course of sepsis, the marked immunosuppressive state requires interventions that can stimulate the immune response. IL-7, which is critical for T-cell development and function, enhances immunity by increasing the expression of cell adhesion molecules, facilitating leukocyte trafficking to sites of infection [33]. Granulocyte macrophage-colony stimulating factor (GM-CSF) restores monocyte function and shortens the duration of mechanical ventilation and length of ICU stay [34].

\section{Extracorporeal blood purification techniques}

In the last few decades, many studies have evaluated the clinical and biological effects of different extracorporeal blood purification techniques in sepsis-associated AKI. The use of RRT in septic patients has been evaluated both for renal support and immunomodulation. Traditional RRT indications are uremia, metabolic disturbances, fluid overload and electrolyte derangements. However, several authors have proposed the use of different RRT techniques to remove inflammatory mediators potentially involved in AKI and distant organ damage. Large clinical trials have suggested that, in septic AKI, early initiation of RRT and the use of continuous and not intermittent strategies are associated with a better hemodynamic profile and outcome. However, the timing of RRT initiation remains heterogeneous in clinical practice and is not yet definitely supported by consistent scientific evidence. Excessive delays in RRT initiation have been associated with higher mortality rates and with worsening of renal function. However, the only published randomized controlled trial available showed no significant differences in renal outcomes or patient survival between early and late initiation of dialysis [11]. The ongoing IDEAL-ICU study (Initiation of Dialysis EArly versus Late in the Intensive Care Unit) will help define the optimal timing of RRT in septic AKI patients [35]. The use of continuous renal replacement therapies (CRRT) in septic AKI is still preferred because of their relationship with better hemodynamic tolerability and with enhanced renal recovery compared to intermittent modalities. In a retrospective study examining patients undergoing continuous therapies versus daily hemofiltration, Sun et al. suggested that patients undergoing continuous veno-venous hemofiltration $(\mathrm{CVVH})$ had a significant improvement in renal function, although the all-cause mortality rates were similar at 60 days [36].

Another relevant issue in RRT for sepsis-associated AKI is the dose of renal support, which has been evaluated in different randomized clinical trials since the initial data from the Vicenza study in which a dose of 
$35 \mathrm{ml} / \mathrm{kg} / \mathrm{h}$ was associated with a better survival of AKI patients, in particular in the presence of sepsis [37]. Unfortunately, subsequent studies, including the RENAL and the Acute Renal Failure Trial Network (ATN) trials, did not confirm these encouraging results $[38,39]$. The RENAL study [38] compared a dose of $25 \mathrm{vs.} 40 \mathrm{ml} / \mathrm{kg} / \mathrm{h}$ in continuous veno-venous hemodiafiltration (CVVHDF), whereas the ATN study [39] compared 20 vs. $35 \mathrm{ml} / \mathrm{kg} / \mathrm{h}$ in 3 times/week intermittent or continuous dialysis. Both studies showed that an increased intensity of the RRT dose had no beneficial effect on outcome (mortality was the primary endpoint of both studies). However, in the RENAL study, a post-hoc analysis of septic patients showed a tendency toward a reduction in mortality rate in the group of patients treated with the higher-intensity approach $(40 \mathrm{ml} / \mathrm{kg} / \mathrm{h})$ [38]. Recently, the multicenter randomized controlled trial, IVOIRE (hIgh VOlume in Intensive caRE) evaluated the impact of high-volume hemofiltration (HVHF) on 28-day mortality in critically ill patients with septic shock and AKI. In this study, the authors did not observe a reduction in 28-day mortality or an early improvement in hemodynamic profile or organ function using $\mathrm{HVHF}$ at $70 \mathrm{ml} / \mathrm{kg} / \mathrm{h}$ compared to standard-volume hemofiltration at $35 \mathrm{ml} / \mathrm{kg} / \mathrm{h}$ [40]. Even though the above-mentioned studies led to negative results in sepsis-associated AKI, they have helped define an optimal dose of dialysis, taking into consideration the difference between prescribed and delivered doses.

As stated before, the application of RRT in patients with septic AKI also has the purpose of increasing the clearance ofinflammatory mediators involved in tissue injury. Over the years, different extracorporeal techniques have been developed leadingto a wide range of possible therapeutic approaches.

1. Standard RRT techniques (CVVH, CVVHD and CVVHDF) using high molecular flow membranes (HFM) or membranes with enhanced adsorption capacity. HFM have an average cut-off value of approximately $30-40 \mathrm{kDa}$ and are capable of eliminating significant amounts of inflammatory mediators including chemokines and cytokines in the middle-molecular weight category. Adsorptive membranes, such as polymethyl methacrylate (PMMA) and AN69ST, have also been used to enhance endotoxin and cytokine clearance and some clinical trials are underway in septic patients with AKI [41]. However, we must emphasize that these inflammatory mediators have a very high generation rate: for this reason, studies using CVVH failed to show any significant modulation of plasma levels of different cytokines [41].
2. Convection-based high-volume techniques (HVHF) are defined by a flow rate of more than $35 \mathrm{ml} / \mathrm{kg} / \mathrm{h}$. Use of HVHF was the subject of a recent Cochrane review [11]: selected trials comparing HVHF with a standard dialysis dose did not show any improvement in patient outcomes. Despite a reported increase in hemodynamic stability and the absence of relevant adverse effects, these studies did not support a strong recommendation for the use of HVHF in critically ill patients with severe sepsis and septic shock. Furthermore, the application of HVHF may potentially cause increased clearance of antibiotics and other drugs, electrolyte disturbances and depletion of micronutrients, which may all lead to a less favorable outcome.

3. High cut-off (HCO) membranes: these membranes are porous enough to achieve the removal of larger molecules $(30-60 \mathrm{kDa})$ mainly by diffusion. Several studies showed benefits of using HCO therapy, such as improved immune cell function, removal of inflammatory cytokines, and a reduction in catecholamine dosage. An undesired effect is albumin loss, which can be attenuated by albumin replacement or by using $\mathrm{HCO}$ membranes in a diffusive and not convective manner [42].

4. Hemoperfusion, hemoadsorption and plasmaadsorption: these techniques involve placement of a sorbent, often a resin, in direct contact with blood or plasma through an extracorporeal circuit. Most of these devices are designed to combine the adsorption strategy with standard RRT. The biocompatibility of these devices is the main limitation for their use and thrombocytopenia and bleeding risk are the most relevant adverse effects [43].

Polymyxin B (PMX-B) is a cationic polypeptide antibiotic with activity against Gram-negative bacteria and high affinity for endotoxin, but its intravenous use has been limited due to the wellknown nephrotoxicity and neurotoxicity. PMX-B has been fixed and immobilized onto polystyrene fiber in a hemoperfusion column cartridge that allows endotoxin removal without toxic effects [44]. The main mechanism of action is through removal of circulating endotoxin, although its effects are likely pleiotropic including the entrapment of inflammatory cells, such as monocytes and neutrophils, and the clearance of cytokines TNF- $\alpha$ and IL-6 with a consequent reduction in the intracellular mechanisms of apoptosis. Cruz et al. [44] published a meta-analysis showing that PMX-B hemoperfusion used in patients with severe sepsis led to an improvement in hemodynamics as measured by mean arterial pressure as well as in oxygenation. These results were observed in the Early Use of 
Polymyxin Hemoperfusion in Abdominal Septic shock (EUPHAS) trial in Europe that confirmed preliminary data coming from the Japanese experience [45]. However, the sample size of these studies was small and confirmation of these clinical benefits in larger studies is still awaited. The first randomized, controlled, diagnosticdirected and theragnostic trial, named EUPHRATES (Evaluating the Use of Polymyxin B Hemoperfusion in a Randomized controlled trial of Adults Treated for Endotoxemia and Septic shock) is still ongoing in the US and Canada [46].

The LPS adsorber is a medical device designed for extracorporeal use, which contains a series of porous polyethylene plates coated with a peptide specific to endotoxin. Yaroustovsky et al. compared the LPS adsorber and PMX-B hemoperfusion in patients with Gram-negative sepsis and reported no significant differences in outcome (small number of enrolled patients) [47].

CytoSorb is a highly adsorptive and biocompatible polymer able to remove multiple inflammatory mediators from the bloodstream. Animal studies have elegantly shown that therapeutic apheresis using CytoSorb can restore chemokine gradients toward infected tissue and away from healthy organs through a sort of leukocyte trafficking control [48]. Coupled plasma filtration adsorption (CPFA) is an extracorporeal treatment based on non-specific adsorption of cytokines and other pro-inflammatory mediators onto a specially designed resin cartridge that is in direct contact with filtered plasma. This system is coupled in series with a standard RRT circuit. Some studies have shown interesting results concerning an improvement in hemodynamics, microvascular derangement and respiratory parameters during the course of CPFA [49].

5. The existing extracorporeal techniques are mainly based on plasma filtration and consequently on substitution of the glomerular function of the kidney. However, standard RRT techniques did not allow some specific functions of transport, metabolic and endocrine activities of tubular epithelial cells to be replaced. To overcome this limitation, Tumlin and coworkers developed a renal assist device (RAD) using a polysulfone filter containing living kidney tubular epithelial cells coupled with a conventional RRT circuit. These authors validated this bioengineered device in several in vivo models demonstrating that septic animals affected by AKI and treated with the RAD maintained reabsorption of $\mathrm{K}^{+}, \mathrm{HCO}^{-}$, and glucose, as well as the excretion of ammonia and normal levels of 1,25-OH-vitamin D3 [50]. Moreover, the RAD modulates systemic inflammation by regulating circulating levels of several cytokines. Based on these preclinical studies, a randomized controlled trial was performed and showed that the RAD induced about a $50 \%$ reduction in 180-day mortality when compared to standard CVVH [50]. Moreover, the RAD was able to modulate plasma levels of several cytokines, including G-CSF, IL-6, and IL-10, thus improving both the early SIRS and late immunoparalysis typical of septic AKI. Of interest, the clinical trial with the RAD was prematurely interrupted because the investigators also observed a significant decrease in mortality using a sham cartridge not containing viable tubular cells. Following this observation, the same authors developed the so-called selective cytophoretic device (SCD) able to sequestrate activated leukocytes within the membrane, inhibiting the release of harmful mediators. Preliminary studies indicated that, when coupled to a standard hemofilter, SCD reduced mortality and dialysis dependence in septic patients. However, the beneficial effects of SCD were observed only when citrate but not heparin was used as the anticoagulant strategy [51]. This finding emphasizes the potential anti-inflammatory properties of citrate, which may at least in part explain the data on a reduction in mortality in clinical trials in ICU patients subjected to RRT with citrate [51].

\section{Stem cell therapies}

Stem cell-based therapies have been proposed in almost all fields of medicine with controversial success. One of the major limitations of this approach comes from the host immune reaction as well as from the possibility of cell dysplasia and tumorigenesis or other maladaptive responses including maldifferentiation, tissue fibrosis, calcification and innate immunity dysregulation. For these reasons, despite a large number of experimental studies, effective clinical results are still lacking, even in the field of sepsis [52].

Bone marrow-derived stem cells of both mesenchymal and hematopoietic origin have been extensively studied in different experimental models of AKI. Mesenchymal stem cells are a heterogeneous population that can be isolated from a variety of adult tissues of mesodermal origin, including bone marrow, adipose tissue, placenta, umbilical cord, dental pulp and synovia. For their regenerating and immune-modulatory effects, mesenchymal stem cells have been tested in several experimental models of acute tissue injury models including AKI and kidney transplantation [52]. Mesenchymal stem cells are able to sense inflammation through the expression of cytokine receptors and 
adhesion molecules. Mesenchymal stem cells can induce M2 macrophages as well as T-regulatory cells [52]. Mesenchymal stem cell-related anti-inflammatory M2 macrophages have also been induced in sepsis models. Moreover, mesenchymal stem cells improved monocyte and neutrophil phagocytosis and reduced bacterial load in different organs (peritoneal cavity, blood, spleen and liver) [53].

Endothelial progenitor cells are circulating committed cells involved in vascular regeneration processes. The therapeutic use of endothelial progenitor cells may be of particular interest in sepsis because of their major role in protection from endothelial dysfunction. Moreover, sepsis is associated with a severe depletion in the endothelial progenitor cell circulating pool and this impairment independently correlates with a worse outcome [54].

Recent studies have suggested that most of the beneficial effects of mesenchymal stem cell and endothelial progenitor cell infusions are observed in the absence of cell engraftment within injured tissues [55]. In addition, the infusion of stem cell supernatants induced a protective effect similar to that observed after whole cell transplantation [55]. Taken together, these results suggest that endocrine/paracrine factors promote the regenerative effects of stem cells. In this setting, recent studies have demonstrated the potential role of microvesicles released from stem cells in tissue regeneration following AKI. Microvesicles are cell fragments involved in cell-tocell communication that are able to shuttle different RNA subsets (mRNA and microRNA), proteins and lipids. In different experimental AKI models, microvesicle administration was associated with improved renal function, histological lesions and survival, preventing the progression toward end-stage CKD [56]. These regenerative effects were partially due to the epigenetic reprogramming of target injured renal cells through the horizontal transfer of mRNA and microRNA [57].

\section{Conclusion}

Experimental and clinical studies have proven the presence of a detrimental cross-talk between sepsis, the systemic inflammatory response to infection, and the development of AKI. Sepsis represents the main cause of renal dysfunction in critically ill patients admitted to the ICU. From a pathogenic point of view, it has been shown that the damage of renal endothelial and tubular epithelial cells during sepsis occurs in the absence of evident signs of tissue hypoperfusion. Indeed, septic AKI develops in the presence of a normal or even increased RBF. These results suggest that the pathogenic mechanisms of septic AKI are associated with the detrimental activity of circulating pro-inflammatory and pro-apoptotic mediators that directly bind to renal resident cells. In particular, the onset of apoptosis, necrosis, necroptosis, autophagy, mitophagy and cell cycle arrest has been identified as the main mechanism of tubular injury during sepsis. Early identification of sepsis-associated AKI using new biomarkers ('omics' technologies) may improve patient outcomes. Moreover, new therapeutic strategies based on pharmacological agents, extracorporeal blood purification techniques and stem cell infusion have been developed and exciting clinical results are expected in the next few years.

\section{Competing interests}

The authors declare that they have no competing interests.

\section{Authors' contributions}

SD edited the session on the pathogenesis of sepsis-associated AKI, MM edited the session of experimental therapies, VC contributed to both parts and supervised the paper.

\section{Declarations}

Publication of this supplement was funded by the corresponding author's institution.

\section{Author details}

${ }^{1}$ Department of Medical Science, Turin University, AOU Città Della Salute e Della Scienza, Turin, Italy. ${ }^{2}$ ASLCN1, S.C. Nefrologia e Dialisi, Dipartimento Area Medica, Cuneo, Italy. ${ }^{3}$ Department of Translational Medicine, University of Eastern Piedmont, "Maggiore della Carità" University Hospital, Novara, Italy.

Published online: 15 March 2016

\section{References}

1. Gomez H, Ince C, De Backer D, et al. A unified theory of sepsis-induced acute kidney injury: inflammation, microcirculatory dysfunction, bioenergetics, and the tubular cell adaptation to injury. Shock. 2014;41:3-11.

2. Glodowski SD, Wagener $\mathrm{G}$, et al. New insights into the mechanisms of acute kidney injury in the intensive care unit. J Clin Anesth. 2015;27:175-80.

3. Murugan R, Karajala-Subramanyam V, Lee M, et al. Genetic and Inflammatory Markers of Sepsis (GenIMS) Investigators: acute kidney injury in non-severe pneumonia is associated with an increased immune response and lower survival. Kidney Int. 2010;77:527-35.

4. Lerolle N, Nochy D, Guérot E, et al. Histopathology of septic shock induced acute kidney injury: apoptosis and leukocytic infiltration. Intensive Care Med. 2010;36:471-8.

5. Di Giantomasso D, May CN, Bellomo R. Vital organ blood flow during hyperdynamic sepsis. Chest. 2003;124:1053-9.

6. Brenner M, Schaer GL, Mallory DL, Suffredini AF, Parrillo JE. Detection of renal blood flow abnormalities in septic and critically ill patients using a newly designed indwelling thermodilution renal vein catheter. Chest. 1990;98:170-9.

7. Mariano F, Cantaluppi V, Stella M, et al. Circulating plasma factors induce tubular and glomerular alterations in septic burns patients. Crit Care. 2008;12:R42.

8. Di Giantomasso D, Morimatsu H, May CN, Bellomo R. Intrarenal blood flow distribution in hyperdynamic septic shock: Effect of norepinephrine. Crit Care Med. 2003;31:2509-13.

9. Holthoff JH, Wang Z, Seely KA, Gokden N, Mayeux PR. Resveratrol improves renal microcirculation, protects the tubular epithelium, and prolongs survival in a mouse model of sepsis-induced acute kidney injury. Kidney Int. 2012;81:370-8.

10. Legrand M, Dupuis C, Simon C, et al. Association between systemic hemodynamics and septic acute kidney injury in critically ill patients: a retrospective observational study. Crit Care. 2013;17:R278.

11. Borthwick EMJ, Hill CJ, Rabindranath KS, Maxwell AP, McAuley DF, Blackwood B (2013) High-volume haemofiltration for sepsis. Cochr Database Syst Rev; CD008075. doi:10.1002/14651858.CD008075.pub2. 
12. Taniguchi T, Kurita A, Kobayashi K, Yamamoto K, Inaba H. Dose- and timerelated effects of dexmedetomidine on mortality and inflammatory responses to endotoxin-induced shock in rats. J Anesth. 2008;22:221-8.

13. Boomer JS, To K, Chang KC, et al. Immunosuppression in patients who die of sepsis and multiple organ failure. JAMA. 2011;306:2594-605.

14. Cantaluppi V, Quercia AD, Dellepiane S, Ferrario S, Camussi G, Biancone L. Interaction between systemic inflammation and renal tubular epithelial cells. Nephrol Dial Transplant. 2014;29:2004-11.

15. Cho E, Lee JH, Lim HJ, et al. Soluble CD25 is increased in patients with sepsis-induced acute kidney injury. Nephrol Carlton Vic. 2014;19:318-24.

16. Panacek EA, Marshall JC, Albertson TE, et al. Efficacy and safety of the monoclonal anti-tumor necrosis factor antibody F(ab')2 fragment afelimomab in patients with severe sepsis and elevated interleukin-6 levels. Crit Care Med. 2014;32:2173-82.

17. Yang R, Wang X, Liu D, Liu S. Energy and oxygen metabolism disorder during septic acute kidney injury. Kidney Blood Press Res. 2014;39:240-51.

18. Gocze I, Koch M, Renner P, et al. Urinary biomarkers TIMP-2 and IGFBP7 early predict acute kidney injury after major surgery. PLoS One. 2015;10: e0120863.

19. Kalakeche R, Hato T, Rhodes G, et al. Endotoxin uptake by S1 proximal tubular segment causes oxidative stress in the downstream S2 segment. J Am Soc Nephrol. 2011;22:1505-16.

20. Naito M, Bomsztyk K, Zager RA. Endotoxin mediates recruitment of RNA polymerase II to target genes in acute renal failure. J Am Soc Nephrol. 2008;19:1321-30.

21. Lentini P, de Cal M, Clementi A, D'Angelo A, Ronco C. Sepsis and AK in ICU patients: the role of plasma biomarkers. Crit Care Res Pract. 2012;2012:856401

22. Vanmassenhove J, Glorieux G, Lameire $N$, et al. Influence of severity of illness on neutrophil gelatinase-associated lipocalin performance as a marker of acute kidney injury: a prospective cohort study of patients with sepsis. BMC Nephrol. 2015;16:18.

23. Si Nga H, Medeiros P, Menezes P, Bridi R, Balbi A, Ponce D. Sepsis and AKI in Clinical Emergency Room Patients: The Role of Urinary NGAL. BioMed Res Int. 2015;2015:413751.

24. Su L, Feng L, Zhang J, et al. Diagnostic value of urine sTREM-1 for sepsis and relevant acute kidney injuries: a prospective study. Crit Care. 2011;15:R250.

25. Bagshaw SM, Langenberg C, Haase M, Wan L, May CN, Bellomo R. Urinary biomarkers in septic acute kidney injury. Intensive Care Med. 2007:33:1285-96.

26. Sood MM, Shafer LA, Ho J, et al. Cooperative Antimicrobial Therapy in Septic Shock (CATSS) Database Research Group: Early reversible acute kidney injury is associated with improved survival in septic shock. J Crit Care. 2014;29:711-7.

27. Opal SM, Laterre P-F, Francois B, et al. ACCESS Study Group: Effect of eritoran, an antagonist of MD2-TLR4, on mortality in patients with severe sepsis: the ACCESS randomized trial. JAMA. 2013;309:1154-62

28. Swaminathan S, Rosner MH, Okusa MD. Emerging therapeutic targets of sepsis-associated acute kidney injury. Semin Nephrol. 2015;35:38-54.

29. Pickkers P, Heemskerk S, Schouten J, et al. Alkaline phosphatase for treatment of sepsis-induced acute kidney injury: a prospective randomized double-blind placebo-controlled trial. Crit Care. 2012;16:R14.

30. Sardinha J, Kelly MEM, Zhou J, Lehmann C. Experimental cannabinoid 2 receptor-mediated immune modulation in sepsis. Mediators Inflamm. 2014;2014:978678.

31. Rosas-Ballina M, Olofsson PS, Ochani $M$, et al. Acetylcholine-synthesizing $T$ cells relay neural signals in a vagus nerve circuit. Science. 2011;334:98-101.

32. Sharfuddin AA, Sandoval RM, Berg DT, et al. Soluble thrombomodulin protects ischemic kidneys. J Am Soc Nephrol. 2009;20:524-34.

33. Unsinger J, McGlynn M, Kasten KR, et al. IL-7 promotes T cell viability, trafficking, and functionality and improves survival in sepsis. J Immunol. 2010;184:3768-79.

34. Meisel C, Schefold JC, Pschowski R, et al. Granulocyte-macrophage colonystimulating factor to reverse sepsis-associated immunosuppression: a double-blind, randomized, placebo-controlled multicenter trial. Am J Respir Crit Care Med. 2009;180:640-8.

35. Barbar SD, Binquet $C$, Monchi M, Bruyère R, Quenot JP. Impact on mortality of the timing of renal replacement therapy in patients with severe acute kidney injury in septic shock: the IDEAL-ICU study (initiation of dialysis early versus delayed in the intensive care unit): study protocol for a randomized controlled trial. Trials. 2014;15:270.

36. Sun Z, Ye H, Shen X, Chao H, Wu X, Yang J. Continuous venovenous hemofiltration versus extended daily hemofiltration in patients with septic acute kidney injury: a retrospective cohort study. Crit Care. 2014;18:R70.

37. Ronco C, Bellomo R, Homel P, et al. Effects of different doses in continuous veno-venous haemofiltration on outcomes of acute renal failure: a prospective randomised trial. Lancet. 2000;356:26-30.

38. Bellomo R, Cass A, Cole L, et al. Intensity of continuous renal-replacement therapy in critically ill patients. N Engl J Med. 2009:361:1627-38.

39. Palevsky PM, Zhang JH, O'Connor TZ, et al. Intensity of renal support in critically ill patients with acute kidney injury. N Engl J Med. 2008;359:7-20.

40. Joannes-Boyau O, Honore PM, Perez P, et al. High-volume versus standard volume haemofiltration for septic shock patients with acute kidney injury (IVOIRE study): a multicenter randomized controlled trial. Intensive Care Med. 2013;39:1535-46.

41. Honore PM, Jacobs R, Joannes-Boyau O, et al. Newly designed CRRT membranes for sepsis and SIRS - a pragmatic approach for bedside intensivists summarizing the more recent advances: a systematic structured review. ASAIO J. 2013;59:99-106.

42. Haase M, Bellomo R, Baldwin I, et al. Hemodialysis membrane with a high-molecular-weight cutoff and cytokine levels in sepsis complicated by acute renal failure: a phase 1 randomized trial. Am J Kidney Dis. 2007;50:296-304.

43. Winchester JF, Kellum JA, Ronco C, et al. Sorbents in acute renal failure and the systemic inflammatory response syndrome. Blood Purif. 2003;21:79-84.

44. Cruz DN, Perazella MA, Bellomo R, et al. Effectiveness of polymyxin B-immobilized fiber column in sepsis: a systematic review. Crit Care. 2007;11:R47.

45. Cruz DN, Antonelli M, Fumagalli R, et al. Early use of polymyxin B hemoperfusion in abdominal septic shock: the EUPHAS randomized controlled trial. JAMA. 2009;301:2445-52.

46. Klein DJ, Foster D, Schorr CA, Kazempour K, Walker PM, Dellinger RP. The EUPHRATES trial (Evaluating the Use of Polymyxin B Hemoperfusion in a Randomized controlled trial of Adults Treated for Endotoxemia and Septic shock): study protocol for a randomized controlled trial. Trials. 2014;15:218

47. Yaroustovsky M, Abramyan M, Popok Z, et al. Preliminary report regarding the use of selective sorbents in complex cardiac surgery patients with extensive sepsis and prolonged intensive care stay. Blood Purif. 2009:28:227-33.

48. Peng Z-Y, Bishop JV, Wen X-Y, et al. Modulation of chemokine gradients by apheresis redirects leukocyte trafficking to different compartments during sepsis, studies in a rat model. Crit Care. 2014;18:R141.

49. Livigni S, Bertolini G, Rossi C, et al. Efficacy of coupled plasma filtration adsorption (CPFA) in patients with septic shock: A multicenter randomised controlled clinical trial. BMJ Open. 2014;4:e003536.

50. Tumlin J, Wali R, Williams W, et al. Efficacy and safety of renal tubule cell therapy for acute renal failure. J Am Soc Nephrol. 2008;19:1034-40.

51. Tumlin JA, Chawla L, Tolwani AJ, et al. The effect of the selective cytopheretic device on acute kidney injury outcomes in the intensive care unit: a multicenter pilot study. Semin Dial. 2013;26:616-23.

52. Camussi G, Cantaluppi V, Deregibus MC, Gatti E, Tetta C. Role of microvesicles in acute kidney injury. Contrib Nephrol. 2011;174:191-9.

53. Németh $\mathrm{K}$, Leelahavanichkul A, Yuen PST, et al. Bone marrow stromal cells attenuate sepsis via prostaglandin E(2)-dependent reprogramming of host macrophages to increase their interleukin-10 production. Nat Med. 2009;15:42-9.

54. Cribbs SK, Sutcliffe DJ, Taylor WR, et al. Circulating endothelial progenitor cells inversely associate with organ dysfunction in sepsis. Intensive Care Med. 2012;38:429-36.

55. Camussi G, Deregibus MC, Bruno S, et al. Exosome/microvesicle-mediated epigenetic reprogramming of cells. Am J Cancer Res. 2011;1:98-110.

56. Bruno S, Grange C, Collino F, et al. Microvesicles derived from mesenchymal stem cells enhance survival in a lethal model of acute kidney injury. PLoS One. 2012;7:e33115

57. Cantaluppi V, Medica D, Mannari C, et al. Endothelial progenitor cell-derived extracellular vesicles protect from complement-mediated mesangial injury in experimental anti-Thy1.1 glomerulonephritis. Nephrol Dial Transplant. 2015;30:410-22. 